For submission to: Contemporary Nurse Special Issue, “Advances in Communication, Technology, and Social Media in Nursing”

\title{
"We don't even have Wi-Fi": A descriptive study exploring current use and availability of communication technologies in residential aged care
}

Professor Wendy Moyle ${ }^{\mathrm{a}, \mathrm{b}^{*}}$ PhD $\underline{\text { w.moyle@griffith.edu.au }}$

Dr. Cindy Jones ${ }^{\mathrm{a}, \mathrm{b}}$ PhD C.Jones@griffith.edu.au

Ms. Jenny Murfield ${ }^{\mathbf{a}}$ BSc(Hons) j.murfield@griffith.edu.au

Ms. Toni Dwan ${ }^{\mathrm{a}, \mathrm{c}}$ B Psych(Hons) t.dwan@griffith.edu.au

Professor Tamara Ownsworth ${ }^{\text {a,c }}$ PhD t.ownsworth@griffith.edu.au

${ }^{a}$ Menzies Health Institute QLD, Griffith University, Nathan, Brisbane, Queensland, Australia ${ }^{\text {b} S c h o o l ~ o f ~ N u r s i n g ~ a n d ~ M i d w i f e r y, ~ N a t h a n ~ C a m p u s, ~ G r i f f i t h ~ U n i v e r s i t y, ~ N a t h a n, ~ B r i s b a n e, ~}$ Queensland, Australia

'School of Applied Psychology, Mt Gravatt Campus, Griffith University, Mt Gravatt, Brisbane, Queensland, Australia

Affiliation: The research was conducted at Menzies Health Institute QLD, Griffith University 
${ }^{*}$ Corresponding author: Professor Wendy Moyle, Menzies Health Institute QLD, Griffith University, 2.10 Health Sciences (N48), 170 Kessels Road, Nathan, Brisbane, Queensland, 4111, Australia. Tel: +61 73735 5526; Fax: +61 73735 5431; w.moyle@griffith.edu.au

\section{Acknowledgements}

Thanks are expressed to all staff at participating facilities who gave of their time to take part in the telephone survey.

\section{Funding}

This research received no specific grant from any funding agency in the public, commercial, or not-for-profit sectors. 


\title{
"We don't even have Wi-Fi here": A descriptive study exploring the current use and availability of communication technologies in Australian residential aged care
}

\begin{abstract}
Background: There has been significant growth in digital, robotic, and web-based technologies to aid communication between residents living in residential aged care facilities and their family and friends. However, it is unknown to what extent care practices within facilities accommodate such technologies.
\end{abstract}

Aim: To explore the use and availability of communication technologies for use by residents within residential aged care facilities in Queensland, Australia, as reported by facility staff.

Methods: A descriptive, structured telephone survey was undertaken over a one-week period in November, 2016. Using a systematic random sampling framework, every tenth alphabetically listed facility from a total sample of $n=462$ were telephoned between 9:00 and 17:00, Monday to Friday, and invited to participate. Administrative staff, care managers, nursing staff, or other appropriate employees were eligible to complete the survey. Descriptive statistics were generated from each survey question, and any additional comments were summarised.

Results: Forty-one out of a total of 93 contacted residential aged care facilities completed the survey. The telephone was by far the primary form of communication used by residents to communicate with family and friends ( $n=40 ; 97.6 \%)$. Conversely, the use of web-connection communication software (Skype or similar) was uncommon: nearly a quarter $(n=9 ; 22 \%)$ of facilities stated that no resident used it; $53.7 \%(n=22)$ stated $\leq 5 \%$ of residents used it; and two facilities estimated the highest usage at $25 \%$ of residents. When using web-connection 
communication software, residents typically owned their own devices ( $n=26 / 32 ; 81.3 \%)$. Of those facilities that provided residents with technology for the purpose of communication ( $n=6 / 32 ; 18.8 \%)$, it was common for desktop computers to be located within a communal area, whereas hand-held tablets were kept in a secure office.

Conclusion: Use and availability of communication technologies is limited within residential aged care facilities, highlighting a significant lag in the uptake within the sector.

Keywords: aged care; Australia; barriers; ICT; long-term care; older people; social connection; survey.

\section{Impact statement}

Residential aged care facilities are behind in uptake of modern communication technologies to aid connection between residents, family, and friends. 


\section{Introduction}

Improvements in public health, medicine, and nutrition in developed countries have contributed to people living substantially longer (World Health Organisation, 2011) and, coupled with reduced birth rates, this has led to an aging population. Consequently, the demand for residential aged care services has risen exponentially and, within Australia alone, the number of admissions to residential aged care facilities (RACFs) has nearly doubled over the last twenty years (Willis et al., 2016). For many residents, separation due to geographical dispersion means that family members and friends are unable to visit regularly, and this can leave residents feeling socially disconnected and lonely (Moyle, Kellett, Ballantyne, \& Gracia, 2011).

Meaningful relationships and social connection have been identified as important for wellbeing in older adults (Brownie \& Horstmanshof, 2011; Kang, 2002; Moyle et al., 2011). Given that residents can spend less than 29 minutes in conversations during a six-hour daytime period (Alzheimer's Society, 2007), it is paramount for facilities to have in place communication strategies that enable remote connection with family and friends. Whilst all Australian RACF residents have access to a telephone, which provides them with an easily accessible means of talking with family and friends, many struggle to engage with this remote form of communication. Cognitive impairment, memory loss, and behavioural and psychological symptoms of dementia can make it difficult for the resident to remember their relationship with the caller, and the absence of visual clues - such as seeing the person's face and mannerisms - cannot help them to recall the caller. This can lead to feelings of frustration and distress, and can inadvertently exacerbate the sense of loneliness the telephone call was intended to alleviate. Technology-enabling communication formats may effectively address the challenges posed by traditional telephone calls, with real-time video providing both audio and visual aids for the resident and caller. 
Over the last five years, there has been significant growth in digital smartphones, robotic, and web-based technologies that can be used to connect residents with family and friends. Statistics from the Interactive Advertising Bureau (IAB) Australia (2015) on smartphone and tablet ownership report on the significant upward trend in use of smartphone and tablets in Australia, and a stagnation or decline in desktop and laptops. Common commercially available technologies, such as iPads and web-connection software (e.g., Skype, FaceTime), have been shown to improve sense of connection for RACF residents (Francis, 2012; Risser, 2014), whilst communication via videophones has been reported to increase feelings of social presence and closeness for both the resident and the caller, and provide visual reassurance to family members that the resident is being cared for in the facility (Demiris et al., 2008; Hensel, Parker-Oliver, Demiris, \& Rantz, 2009). More sophisticated, cutting-edge communication technologies, including mobile telepresence technology, have also demonstrated feasibility and efficacy for use in RACFs. Indeed, findings from a pilot study we conducted using the mobile telepresence robot, Giraff - a free-standing, wheel-based mobile robot, with a video-conferencing system that can be controlled by a remote user - revealed improved connection and communication between residents and their families (Moyle et al., 2014). Importantly, however, the robot also provided the added advantage of being able to move around the facility with the resident, make 'social gestures', such as moving the display head up and down - 'nod', and adjustment of the display height from a standing to sitting position, as appropriate.

Given continued and ongoing technological advancements, coupled with the dispersion of many residents' families, and the demonstrated benefits of using modern communication technologies, it is reasonable to assume that RACFs would be starting to accommodate such technologies within their practice for resident use. However, at present, it is unknown what the current levels of use and availability are amongst residents in RACFs. 


\section{Method}

\section{Aim}

The study aimed to explore the current use and availability of communication technologies for use by residents within RACFs in Queensland, Australia. In doing so, the survey sought to identify, through interviews with facility staff: 1) the main communication technology used by RACF residents to connect to their family or friends; and 2) the proportion of RACF residents using web-connection software (i.e., Skype or similar) to connect to family or friends, and whether these technologies were individually owned or provided, and where kept, by the facility.

\section{Design}

A brief, descriptive, structured telephone survey was undertaken via a systematic random sample of RACFs in November, 2016. This design was chosen as it permitted the collection of up-to-date, quantifiable data that, due to the random sampling, could be used to make general, non-causal inferences about technology use in other, similar RACFs. The survey was conducted by telephone, as opposed to face-to-face, to encourage good response rates and reach facilities from across the large study area (Queensland, the second-largest state in Australia).

\section{Sample and setting}

At the time of the study, there were $n=462$ government approved and accredited RACFs located in Queensland, Australia and listed on the Aged Care Guide ${ }^{\circledR}$ directory website (the largest aged care online resource in Australia). All RACFs were potentially eligible for inclusion in the survey. Using a systematic random sampling framework, every tenth facility listed alphabetically on the directory was telephoned, with the next, immediately listed entry 
called if there was no answer, unavailability, or refusal to complete the survey. Due to the survey having limited internal funding, data collection was restricted to a one-week period, or a 10\% sample of facilities ( $n=46)$, whichever was achieved first.

\section{Data collection and analysis}

All RACFs were telephoned between the hours of 9:00 and 17:00, Monday to Friday, by one team member (JM). In all telephone approaches, a standard script was followed that outlined the research to the person who answered the telephone - typically the receptionist - and detailed what type of information was requested (Table 1). This person was given the option of completing the survey themselves, if they could, or was asked to direct the call to either the care manager, a nurse, or other appropriate employee able to provide the information. If the person answering the telephone was unsure if they had the information to complete the survey, a brief outline of the questions was given by the interviewee. This ensured that all respondents competing the survey had the information able to answer every question and that there were no instances of missed questions due to limited knowledge or information. The survey lasted between three to five minutes and, due to the short, closed-response nature of the questions, relied on interviewer notes as opposed to being audio-recorded.

$$
\text { [Insert Table1 about here] }
$$

All verbally-collected survey data were directly entered into a purpose-designed Microsoft Excel (2013) spreadsheet during or immediately after each survey, and then exported into IBM SPSS Statistics for Windows version 24.0 (Armonk, NY: IBM Corp) for analyses. Responses to each question were assigned a numeric value to enable categorisation (i.e., for interviewee role, 1= Management, 2= Nurse, etcetera.). The Australian Standard Geographical Classification - Remoteness Areas (ASGC-RA 2006) (Australian Bureau of 
Statistics, 2006), was used to geographically categorise the location of surveyed RACFs according to five standard categories: major cities; inner regional; outer regional; remote; and; very remote. Basic, descriptive statistics were generated for each survey question, and the few additional qualitative comments - where voluntarily provided - were read and summarised by two team members and used, where appropriate, alongside the quantitative survey data to provide additional context (JM \& TD).

\section{Ethics}

Institutional ethical approval was obtained from Griffith University Human Research Ethics Committee (NRS/2016/861). Verbal informed consent was obtained from all participants immediately prior to commencement of the survey. Names of every facility and participant were voluntarily provided for administrative research purposes. All were de-identified prior to analyses and reporting to ensure anonymity.

\section{Results}

A total of 93 facilities were contacted over a one-week period and, of these, employees from 41 RACFs took part in the survey. This equated to a 44.1 per cent successful response rate, and represented nine per cent of the total of number of RACFs in Queensland, Australia at the time of survey ( $n=462)$. Reasons for non-participation included: no answer at the facility ( $n=23 ; 24.7 \%)$; refusal ( $n=19 ; 20.4 \%)$, with 10 respondents (all administration staff) specifically stating that the aged care organisation Head Office would be more appropriate to complete the survey; and unavailability ( $n=10 ; 10.8 \%)$.

Of the surveyed RACFs, the largest proportion were located in a geographical area classified as a major city ( $n=29 ; 70.7 \%)$. The remaining facilities were located in areas classified as inner or outer regional Queensland ( $n=6 ; 14.6 \%$ for both). No facilities agreed to 
be surveyed in remote or very remote Queensland classification areas (Table 2). Regarding the type of facility employee completing the survey, the majority were administrators $(n=17$; 41.5\%) or members of the senior management team, such as the Care Manager $(n=16 ; 39 \%)$ (Table 3).

[Insert Tables 2 and 3 about here]

The audio-only telephone was reported by 40 out of 41 RACFs as the most common form of communication used by residents to talk with their family and friends ( $n=40 ; 97.6 \%)$. Use of email software was reported by the other RACF as the most common method of communication used by residents $(n=1 ; 2.4 \%)$.

Regarding the proportion of residents using web-connection software (i.e., Skype or similar) to communicate with family or friends, estimations were very low and, indeed, such was the rarity that respondents could individually count and name residents who used webconnection software within their facility (Table 4). Specifically, nearly a quarter $(n=9 ; 22 \%)$ of facilities stated that none of their residents used web-connection software, and a further 53.7 per cent $(n=22)$ estimated it to be used by just five per cent or less of all residents. These facilities were not limited to a geographical area, but were stochastically spread across all three surveyed classification areas. The highest estimated proportion of web-connection communication software usage by residents across facilities was 25\%; estimated by two facilities, both of which were located in inner regional Queensland.

[Insert Table 4 about here]

Of the 32 surveyed facilities with residents using web-connection communication software to connect to family and friends, it was typical for these technologies to be privately owned by the individual residents $(n=26 ; 81.3 \%)$. One Care Manager voluntarily noted that 
their facility “don’t even have Wi-Fi, so residents have to provide their own to be able to use internet-based technologies.” The remaining 18.8\% $(n=6)$ of facilities reported that they provided shared technology, in addition to residents owning their own devices. In instances when the facility provided desktop computers, these were chiefly located within a communal area ( $n=5$ out of 6 ). However, when hand-held tablets (i.e., iPads) were provided by the facility, all were kept in Diversional Therapist offices and brought out "as and when” ( $n=3$ out of 6) required. A surveyed Care Manager qualified the reason for the secure keeping of devices by stating that "these things tend to go astray, so they need to be kept by the DT [Diversional Therapist].”

\section{Discussion}

In this brief, small-scale, descriptive telephone survey, we found that both use and availability of electronic communication technologies is currently limited within RACFs. In the facilities we surveyed, audio-only telephones were still the primary form of communication technology used by residents to connect with family and friends. Whilst acknowledging that telephones provide a familiar and easily accessible means of maintaining residents' social connection - known to be important in reducing feelings of isolation and contributing to improved wellbeing (Brownie \& Horstmanshof, 2011; Kang, 2002; Moyle et al., 2011) - many residents, particularly those with cognitive impairment, find that telephones are frustrating to use as a means of communication, as they rely solely on audio stimuli to connect the person's voice to an individual. Web-connection software, such as commonlyaccessible programs Skype and FaceTime, whilst likely to be unfamiliar to residents and requiring assistance from staff to initiate connection of the call, may help to overcome this particular challenge by providing real-time video with both audio and visual aids for the resident and caller. However, we found that uptake of web-connection software was limited 
in surveyed facilities, with three-quarters estimating that only five percent or less of their residents used this type of technology. Given the prolific use of web-communication software within modern life following the significant adoption of smartphone use, these findings raise important questions about what may be prohibiting the uptake of advanced communication technologies within RACFs that should be determined in future research.

One important barrier identified in previous research relates to the reluctant attitude of staff to integrate technology within care (Sävenstedt, Sandman, \& Zingmark, 2006), as well as a presumption that residents would not want to use the technology over more traditional means (Sävenstedt et al., 2006). Although not specifically explored in our survey, some of the voluntary comments from contacted facilities suggest that this may still be an issue. Some facilities declining to participate in the research stated that they did not "do much" with technology because of the nature of the clientele they provided care for (complex, high needs, and many with dementia), and seemed generally unsure of the relevance of technology use to their facility. In these instances, we were typically asked to call the aged care organisation Head Office to obtain the information. Yet, research has shown that the use of video-based communication is particularly advantageous for those with dementia.. At times, videophone communication between residents with dementia and their family member can enable a more focused and better quality conversation than even face-to-face discussions (Sävenstedt, Brulin, \& Sandman, 2003), whilst more cutting-edge mobile telepresence robots can improve engagement and reduce social isolation, and also permit movement around the facility (Moyle et al., 2014). To encourage uptake, more evidence from clinical trials on the efficacy of communication technologies with the RACF population is needed. This evidence can then be used to inform changes in care practice guidelines, and also the development of education and training initiatives for all levels of facility staff, as well as families and residents, on the 
benefits of remote communication. Education on the general inclusion of information technology communication (ICT) within facilities is also needed.

Another key issue likely contributing to the limited uptake of advanced technologies within our surveyed RACFs relates to the lack of provision within facilities to accommodate web-connection communication. For one surveyed facility, located in an area classified as a major city, there was no internet access or Wi-Fi at the site. We suspect that, although mindful of overstating beyond the limits of our data, lack of adequate internet-connection may have been a more common issue had we been able to survey RACFs in remote and very remote parts of Queensland. Nevertheless, lack of the required internet connection needed to access modern communication technologies is evidently a significant barrier to uptake. The current roll-out of the Australian national broadband network ${ }^{\mathrm{TM}}$, due for completion in 2020, will, hopefully, provide a nation-wide fast and reliable internet service, and go some way to addressing this issue. However, even when access is available, it is also important for facilities to spend some time in planning and researching their facility-wide Wi-Fi network. The spread-out, often 'rabbit-warren-like' design of facilities requires careful consideration about the number and location of access points needed to achieve the best Wi-Fi coverage (Bender, 2013). Similarly, it is important for researchers testing new technologies in intervention studies to consider internet connection issues within facilities, as well as the capacity for facilities with Wi-Fi to permit access. In our own research (Moyle et al., 2014), we were not authorised to connect to the facility’s Wi-Fi due to privacy and safety concerns, and had to use plug-in universal series bus (USB) Wi-Fi adaptors/dongles during the trial of a telepresence robot. This exacerbated connectivity issues (e.g., patchy connection) and hindered the use of the technology to its full capacity. Future research should seek to resolve access issues as a priority, and enter into an open dialogue with facilities at the recruitment stage into how privacy and safety can be addressed. In assisting with this, facilities would 
benefit from employing a trusted Wi-Fi security company that can advise them in creating a visibility architecture within the Wi-Fi network to enable monitoring of the security of the system (Bromley, 2016).

Another issue related to the lack of provision within facilities is a lack of available and accessible technologies. In our survey, we found that residents who engaged in webconnection communication did so on devices that they owned personally. Further, in the few instances where shared technology was provided by facilities for the purpose of webcommunication, only desktop computers were placed in communally accessible areas, whereas hand-held tablets were locked away and used on an ad-hoc basis, given that the devices could get easily misplaced or lost. Communication technology is expensive and this may be one reason why it was uncommon for surveyed facilities to provide shared technology for the purposes of communication between residents and their families. However, many hand-held devices have a locating function (e.g., find my phone), and facilities should be encouraged to enable these on current devices to alleviate some concerns over misplacement. That said, further research is needed to explore additional solutions to this practical issue, as it is equally important that expensive technology is secure, but also openly accessible to all residents.

There are several limitations to this study that are important to consider when interpreting these findings. First, the brief, descriptive nature of the study permits only general inferences about the use of communication technologies in RACFs, and, thus, cannot provide any concrete insight into why use and availability within facilities is at the reported levels. Second, issues with generalisability of the results are acknowledged, particularly in light of a small sample size ( $n=41)$, which was less than 10 per cent of the available sample of RACFs ( $n=462$ ), and the decision to limit data collection to a one-week period in November only, and to the Australian-state of Queensland. Third, whilst we employed a 
systematic random sampling framework, in which every tenth facility listed alphabetically on the Aged Care Guide ${ }^{\circledR}$ directory website was contacted, no RACFs from remote or very remote parts of Queensland agreed to participate. As such, it is possible that our findings are an over-estimation of current use and availability, given the more urban locale of surveyed facilities and their potentially better internet and Wi-Fi accessibility. Future survey work of this nature may benefit from employing a stratified sampling framework, with the five geographic classification areas defined as separate stratums, to ensure the inclusion of facilities from all geographies. Fourth, we recognise that, as this was an on-the-spot telephone survey, responses pertaining to number/proportion of residents are based on estimations and may not be completely accurate, and that survey participation may have been higher had a letter been sent to the RACF Director in advance. Further, given that a range of employees completed the survey, it is possible that the different levels of seniority and access to current information may also have affected the accuracy of the data provided by each facility.

\section{Conclusion and implications for practice}

Overall, our findings suggest that there is a significant lag between the plethora of communication technology available, and the current uptake within the aged care sector. As such, efforts to increase use, availability, and accessibility of technologies are very much needed. Further research into the efficacy of communication technologies for use with this population is required so that use may be encouraged through education and training initiatives for RACFs staff, family and residents on the benefits of ICT for remote communication, as well as training in how to use the technologies. In turn, availability and accessibility of technologies could also be enhanced by encouraging facilities to ensure their internet access is sufficient and appropriately secure to accommodate the technology. Whilst costs may be prohibiting the wider provision of communication technologies for residents 
within facilities, making use of locating functions on hand-held tablets may address some of the concerns regarding lost devices.

\section{Disclosure statement}

No potential conflict of interest was reported by the authors. 


\section{References}

Alzheimer's Society. (2007). Home from home: A report highlighting opportunities for improving standards of dementia care in care homes. Retrieved from https://www.alzheimers.org.uk/site/scripts/download_info.php?fileID=270

Australian Bureau of Statistics. (2006). Australian Standard Geographical Classification Remoteness Areas (ASGC-RA 2006). Retrieved from http://www.doctorconnect.gov.au/internet/otd/publishing.nsf/content/ra-intro

Bender, A. (2013). Case study: Bad WiFi setup cost Adelaide aged care facility. Computerworld. 10 January 2013. Retrieved from https://www.computerworld.com.au/article/446095/case_study_bad_wifi_setup_cost adelaide_aged_care_facility/

Bromley, K. (2016). New thinking needed on aged care network security. Australian Ageing Agenda. 21 October 2016. Retrieved from http://www.australianageingagenda.com.au/2016/10/21/new-thinking-needed-agedcare-network-security/

Brownie, S., \& Horstmanshof, L. (2011). The management of loneliness in aged care residents: An important therapeutic target for gerontological nursing. Geriatric Nursing, 32(5), 318-325. doi:http://dx.doi.org/10.1016/j.gerinurse.2011.05.003

Demiris, G., Oliver, D. R. P., Hensel, B., Dickey, G., Rantz, M., \& Skubic, M. (2008). Use of videophones for distant caregiving: An enriching experience for families and residents in long-term care. Jounal of Gerontological Nursing, 34(7), 50-55.

Francis, N. C. (2012). In touch with iPads. Residents of one nursing home find endless possibilities with the swipe of a finger. Provider. Long-term \& post-acute care. November 2012. Retrieved from 
http://www.providermagazine.com/archives/archives-2012/Pages/1112/In-Touch-

With-iPads.aspx

Hensel, B. K., Parker-Oliver, D., Demiris, G., \& Rantz, M. (2009). A Comparison of VideoBased Resident-Family Communication in a Nursing Home and a Congregate Living Facility. Journal of the American Medical Directors Association, 10(5), 342-347. doi:10.1016/j.jamda.2009.02.006

Interactive Advertising Bureau Australia. (2015). 3rd mobile ratings report - September data, 2015. IAB Australia. Retrieved from https://www.iabaustralia.com.au/research-andresources/research-resources/item/12-research-and-resource/2013-3rd-mobile-ratingsreport-september-2015

Kang, H. (2002). Correlates of social engagement in nursing home residents with dementia. Asian Nursing Research, 6, 75-81.

Moyle, W., Jones, C., Cooke, M., O’Dwyer, S., Sung, B., \& Drummond, S. (2014). Connecting the person with dementia and family: A feasibility study of a telepresence robot. BMC Geriatrics, 14(1), 7. doi:10.1186/1471-2318-14-7

Moyle, W., Kellett, U., Ballantyne, A., \& Gracia, N. (2011). Dementia and loneliness: An Australian perspective. Journal of Clinical Nursing, 20(9-10), 1445-1453. doi:10.1111/j.1365-2702.2010.03549.x

Risser, S. (2014). Technology can build community among residents' families, staff. Providers find new ways to increase family involvement. iAdvance Senior Care. Retrieved from https://www.iadvanceseniorcare.com/article/technology-buildcommunity-residents-families-staff

Sävenstedt, S., Brulin, C., \& Sandman, P. O. (2003). Family members' narrated experiences of communicating via video-phone with patients with dementia staying at a nursing 
home. Journal of Telemedicine and Telecare, 9(4), 216-220.

doi:doi:10.1258/135763303322225544

Sävenstedt, S., Sandman, P. O., \& Zingmark, K. (2006). The duality in using information and communication technology in elder care. Journal of Advanced Nursing, 56(1), 17-25. doi:10.1111/j.1365-2648.2006.03975.x

Willis, E., Price, K., Bonner, R., Henderson, J., Gibson, T., Hurley, J., . . Currie, T. (2016). Meeting residents' care needs: A study of the requirement for nursing and personal care staff. Australian Nursing and Midwifery Federation. Retrieved from http://www.anmf.org.au/documents/reports/National_Aged_Care_Staffing_Skills_Mi x_Project_Report_2016.pdf

World Health Organisation. (2011). Global Health and Aging. National Institute on Aging, National Institutes of Health, U.S. Department of Health and Human Services. Retrieved from http://www.who.int/ageing/publications/global_health.pdf 
Table 1. Survey questions.

1. What is the main communication technology used by residents to connect to their family or friends?

2. What proportion of residents use web-connection software, such as Skype or similar, to connect to family or friends?

3. Of those residents who use web-connection software to connect to family and friends, does the facility provide the technology or does it belong to individual residents?

4. [If the RACF provides communication technology, other than audio phone] Where is the communication technology kept in the facility? 
Table 2. Geographical classification of surveyed residential aged care facilities in Queensland, Australia ( $n=41)$.

\begin{tabular}{lcc}
\hline Geographical classification $^{\mathrm{a}}$ & Frequency $(n)$ & Percentage (\%) \\
\hline Major cities & 29 & 70.7 \\
Inner regional & 6 & 14.6 \\
Outer regional & 6 & 14.6 \\
Remote & 0 & 0.0 \\
Very remote & 0 & 0.0
\end{tabular}

${ }^{a}$ The Australian Standard Geographical Classification - Remoteness Areas (ASGC-RA 2006) (Australian Bureau of Statistics, 2006) 
Table 3. Employment position of survey respondents $(n=41)$.

\begin{tabular}{lcc}
\hline Staff member position & Frequency $(n)$ & Percentage (\%) \\
\hline Administration & 17 & 41.5 \\
Management & 16 & 39.0 \\
Nursing & 5 & 12.2 \\
Personal Care Worker & 2 & 4.9 \\
Diversional Therapist & 1 & 2.4 \\
\hline
\end{tabular}


Table 4. Estimated proportion (\%) of residents using web-connection software, such as Skype or similar $(n=41)$.

\begin{tabular}{lcc}
\hline Estimated proportion & Frequency $(n)$ & Percentage (\%) \\
\hline $0 \%$ & 9 & 22.0 \\
$1-5 \%$ & 22 & 53.7 \\
$6-10 \%$ & 6 & 14.6 \\
$20 \%$ & 2 & 4.9 \\
$25 \%$ & 2 & 4.9 \\
\hline
\end{tabular}

